\title{
Overt palmar surface sweating produces positive component of palmar skin potential responses
}

\author{
Katuo YAMAZAKI \\ School of Human Sciences, Waseda University \\ 2-579-15 Mikajima, Tokorozawa 359-1192, Japan \\ Toshihiko OKAMURA \\ Kagoshima Prefectural College \\ 1-52-1 Shimo-Ishiki, Kagoshima 890-0005, Japan \\ and \\ Noriyoshi TAKASAWA \\ National Research Institute of Police Science \\ 6-3-1, Kashiwanoha, Kashiwa 277-0882, Japan
}

\begin{abstract}
We simultaneously recorded palmar sweating and skin potential responses (SPRs) to determine their relationship. We used a highly sensitive capacitance hygrometer to simultaneously record sweating responses from the palm and SPR by DC. Ten healthy male college students were enrolled as subjects. Stimuli such as continuous short tone burst and hand claps were randomly provided to induce sweating responses and SPR. When a total of 202 SPR waves induced were investigated in relation to sweating responses, sweating responses were observed in all 140 responses in which positive components were included in the SPR waves. However, no sweating responses were observed in 62 responses in which the SPR waves exhibited monophasic negative waves. These results suggest a close relationship between the positive components in SPRs and surface sweating. Our findings have presented a clear answer to the controversy surrounding this problem, and have partially verified Edelberg's poral valve model.
\end{abstract}

Key words: Palmar SPR, Palmar sweating, Surface sweating, SPR positive wave, Edelberg's poral valve model

The question of in which part of the skin the elecrodermal response (EDR) emerges has long attracted the attention of researchers. In particular, several models have been proposed with respect to the mechanism of peripheral occurrence of skin potential responses (SPRs) exhibiting negative waves, diphasic waves, and positive waves. It has been suggested that negative waves which are slow in reaching inertia and positive waves which are quick in reaching inertia

Received Feb. 17, 2001; Accepted Mar. 5, 2001 arise from different origins (Forbes \& Bolles, 1936), and the two-effector hypothesis was proposed. On the basis of the study results suggesting that negative waves originate from the sweat glands and positive waves originate from the dermis (Takagi \& Nakayama, 1959), and the finding that positive components are affected when various cations are applied to the skin (Edelberg, Greiner, \& Burch, 1960), it has been assumed that positive components are an independent phenomenon, and emerge at sites different from negative waves. Research results with respect to 
skin conductance responses (SCRs) and SPRs were obtained, leading to the so-called membrane model for the emergence of EDR (Edelberg, 1972). This model hypothesized the presence of a membrane for the reabsorption of sweat, which regulates the level of hydration in the epidermis, and prevents excessive hydration of the skin surface. However, the presence of this membrane and its innervation have not been anatomically demonstrated to date.

Fowles (1986) carefully reviewed various studies on the mechanism of emergence of EDR, and proposed a single innervation model for the abovementioned two components. The EDR emergence phase introduced in this model is composed of the secretion of sweat, the reabsorption of sodium in the dermal duct, the rise of sweat in the epidermal duct, the movement of sweat across the duct wall into the corneum, and the stretching of the duct at the epidermal level which may occur when sweat secretion is sufficiently strong. The stretching of the duct serves as a trigger for activation of the ductal membrane. When stretching of the duct reaches the triggering level, the permeability across the duct wall rapidly increases, resulting in an elevation of conductance. This model is attractive in that it explains the membrane characteristics of the EDR effector, without assuming different innervation for each process of EDR.

It has been a long time since a close association between palmar sweating and EDR was suggested. However, no consistent conclusions have been drawn because of the lack of appropriate technologies for determining sweating, and differences in the methodology of EDR determination employed. In this study, we simultaneously recorded sweating responses from the palm and SPRs using a recently-developed highlysensitive continuous sweating measuring device, and compared our results with various findings reported previously.

\section{Methods}

The subjects were ten healthy male volunteers aged between 19 and 21 years. Informed consent was obtained from all subjects.

\section{Apparatus}

Palmar sweating was determined using a capacitance hygrometry technique. A capsule covering a skin area of $1 \mathrm{~cm}^{2}$, which was mounted on the left hypothenar eminence, was ventilated with dry nitrogen gas at a rate of $0.3 \mathrm{l} / \mathrm{min}$. The humidity of the effluent nitrogen gas was read with a capacitance hygrometer (Hydrograph AMU-2, Fourtion, Nagoya, Japan) and recorded on the pen-recorder described below (Fig. 1). Capacitance hygrometry has a quicker response and a higher linearity over the full range of relative humidity than resistance hygrometry (Sugenoya \& Ogawa, 1985). The time delay in detecting sweating was minimized by reducing the length of the tube connecting the capsule and humidity sensor.

The SPR was measured with equipment which has stable DC amplifiers and a high input impedance recorder (Toa, EPR-23A, Tokyo, Japan). Two Ag-Ag $\mathrm{Cl}$ Beckman cup electrodes filled with $0.05 \mathrm{M} \mathrm{NaCl}$ in Unibase were attached by means of double stick adhesive collars. One active electrode was placed on the left palm adjacent to the capsule and the reference electrode on a lightly abraded ventral surface of the left forearm. Fig. 2 shows the capsule used to measure the sweating response and SPR recording electrodes.

\section{Procedure}

Each subject was seated in a comfortable chair with his eyes closed in a quiet room and was informed in detail of the nature of the experiment. The electrodes and the capsule were then attached, following which was a 10 min resting period. A series of hand

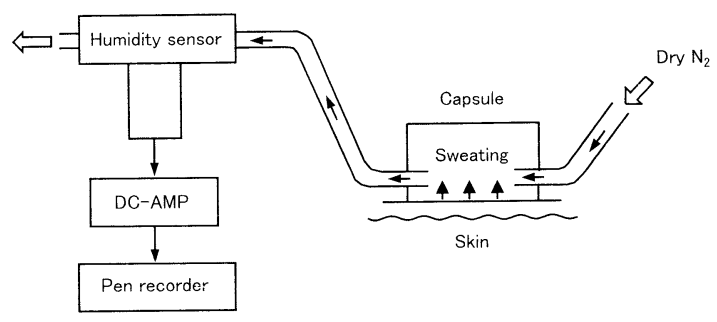

Fig. 1 Diagram of system used to monitor sweating response. 


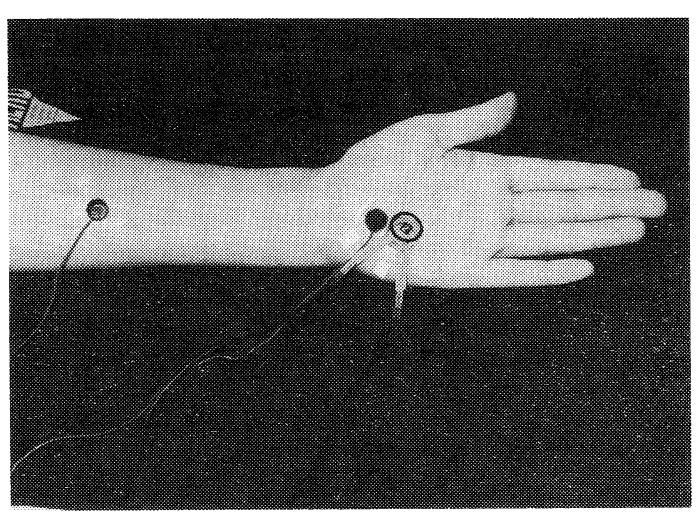

Fig. 2 Capusule used to measure sweating response together with SPR recording electrodes.

claps and bursts of white noise ( $85 \mathrm{~dB}$ SPL intensity and $0.5 \mathrm{~s}$ duration) were administered at random intertrial intervals between 40 and $60 \mathrm{~s}$. The experimenter was seated to the rear of the subject and operated the apparatus. The experimental session lasted approximately $35 \mathrm{~min}$. The room temperature was maintained at 22 to $24{ }^{\circ} \mathrm{C}$. The white noise was administered by a speaker placed $1 \mathrm{~m}$ behind the subject.

\section{Scoring of responses}

SPRs to each stimulus were scored on the basis of the amplitude and wave form observed within 0.5 to $3.0 \mathrm{~s}$ after stimulus onset. SPR negative waves of 0.2 $\mathrm{mV}$ or more were measured from the starting baseline to the peak negative deflection. In diphasic SPRs, an amplitude was measured from the negative to the positive peak. SPR positive waves of $0.2 \mathrm{mV}$ or more were measured from the starting baseline to the peak positive deflection. SPR wave forms were classified into monophasic negative, distorted diphasic, diphasic, and monophasic positive waves. A distorted diphasic wave indicates a wave form in which a negative wave induced by the stimulus shows some small positive notches and then reaches a negative peak, and a wave form in which a negative wave exhibits a sudden deflection, but does not exceed the baseline to turn positive, and increases again on the negative side. Sweating responses of $0.003 \mathrm{mg} / \mathrm{cm}^{2} / \mathrm{min}$ or more were measured from the prestimulus baseline to peak deflection.

\section{Results}

A total of 202 SPRs, including 62 monophasic negative waves, 22 distorted diphasic waves, 98 diphasic waves, and 20 monophasic positive waves, were recorded in response to the above-mentioned stimuli. When all SPRs were paired with sweating responses, it was found that distorted diphasic waves, diphasic waves and monophasic positive waves were all associated with sweating responses (100\%). No sweating response appeared during monophasic negative waves. An example of simultaneous recording of SPRs and palmar sweating is shown in Fig. 3. A significant correlation was obtained between the amplitude of SPR positive components or positive waves, and corresponding sweating responses $(r=0.53 ; F$ $(1,138)=18.82, p<.001)$.

\section{Discussion}

The ventilation method is suited to quantitative measurement of local sweating. The ventilation method is the most common method at present, by which a capsule covering a small area on the skin is ventilated with dry air, and increases in humidity at the site are determined and recorded with a hygrometer. Resistance hygrometry, which is a variation of this method, uses humidity-related changes in electric resistance of hygroscopic elements, and is capable of continuously recording the sweating volume, and accurately detecting subtle variations in sweating. However, a disadvantage of this method is that the responses do not accurately reflect decreases in the sweating volume because the dehumidification rate of the hygroscopic substances is relatively slow. The capacitance hygrometry method using a highly-sensitive apparatus employed in this study is superior to resistance hygrometry in terms of the frequency properties, accuracy in reflecting decreases in humidity, and reliability of the data obtained (Sugenoya \& Ogawa, 1985; Sugenoya, Iwase, Mano, \& Ogawa, 1990).

A close correlation between EDRs and sweating has been suggested by a number of studies. It seems important to present some of these studies for 


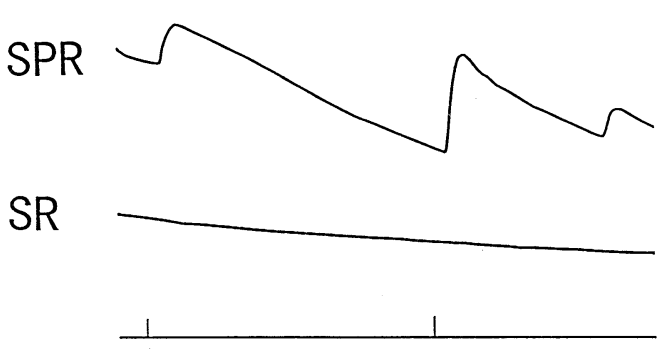

C

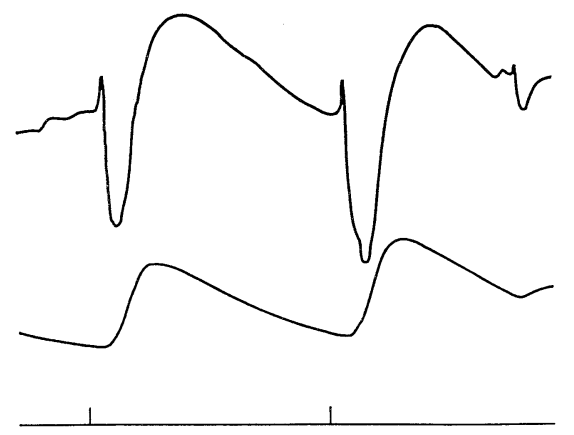

b

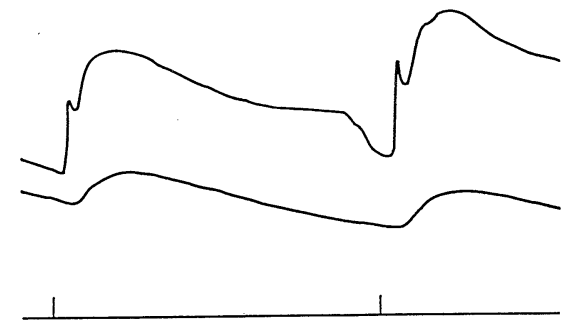

d

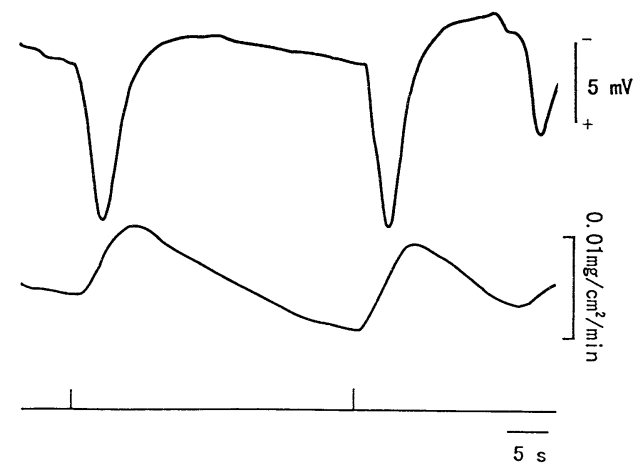

Fig. 3 Simultaneous recording of SPRs and sweating responses(SR).

The notch on the event marker indicates tonal stimulus. Negativity at the active electrode of SPR is indicated by an upward deflection. No sweating response is appeared during monophasic negative waves(a). Sweating responses are always observed during positive component of $\operatorname{SPRs}(b, c, d)$.

interpreting our study results. Reports of the lack of SCRs or SPRs in patients with a congenital absence of sweat glands (Richter, 1927), or patients after peripheral nerve section and sympathetic ganglionectomy (Richter, 1927; Richter \& Woodruff, 1941), and reports which suggest that, similarly to after peripheral nerve section, blocking of the activities of sweat glands by such drugs as atropine eliminates SCR and SPR (Lader \& Montagu, 1962; Venables \& Martin, 1967; Wilcott, 1964) seem to suggest that EDRs do not emerge in the absence of sweat gland activities. Regarding the relationship between EDR and surface sweating, the emergence of negative components in SCRs and SPRs about one second prior to surface sweating has been reported (Darrow, 1932, 1964; Wilcott, 1958, 1962). These findings indicate that negative components in SCRs and SPRs originate from electric activities deep within sweat glands.
There is also a report suggesting a close association between SCRs with a rapid recovery limb and sweating or positive components of SPRs, and that positive SPR waves are more closely associated with sweating than negative SPR waves (Darrow, 1932, 1964).

According to a study on latency in EDRs and latency in surface sweating (Wilcott, 1962), there was almost indistinguishable synchronism between the latency in positive components of SPRs and the latency in surface sweating, in contrast to negative components of SPRs. Moreover, obvious sweating was not observed in some cases during the emergence of SCRs and monophasic negative SPR waves. These findings on EDRs and sweating appear to indicate close associations between these two factors. Wilcott (1962) simultaneously recorded and quantitatively analyzed sweating responses and SPRs, and reported 
correlations of 0.62 to 0.80 between sweating responses and negative SPR waves, and close correlations of 0.81 to 0.95 between sweating responses and positive SPR waves. It is true that the correlation between sweating and positive SPR waves was strong, but some correlations were also noted between sweating and negative SPR waves, and therefore, it is difficult to speculate on the association between sweating and negative or positive components of SPRs from these correlation coefficients.

Several studies have attempted the simultaneous recording of sweating and skin resistance responses (SRRs) which exhibit only monophasic simple variations, and reported correlations of 0.8 to 0.9 between these factors (Adams \& Vaughan, 1965; Wilcott, 1962). These studies suggest a $1: 1$ correlation between the sweating volume and the SRR amplitude. However, another study has reported significant correlations in only four of 12 subjects investigated, failing to observe meaningful correlations in the remaining eight subjects (Edelberg, 1964).

The greatest reason why the past studies have failed to observe obvious associations between the SPR wave forms and sweating, or failed to agree on the relationship between sweating and SRRs or SCRs, may be that sweat-measuring apparatuses with a relatively slow dehumidification rate of hygroscopic substances was used in these studies, as mentioned above. Therefore, the responses observed in these studies may not have accurately reflected the decreases in sweating volume. Highly-sensitive sweat-measuring devices were not available at that time.

In the current study, the emergence of sweating responses was not observed when the SPR waveform showed negative waves. On the other hand, the emergence of sweating responses was confirmed when the SPR waveform exhibited distorted diphasic, diphasic, and positive waves. These results indicate that the SPR negative waves represent electric phenomena deep within the sweat gland, while positive SPR waves and positive components suggest electric phenomena closely associated with surface sweating.
Concerning the physiological mechanism of the emergence of SPRs, Fowles $(1974,1986)$ suggested that negative components emerge while the sweat secreted from the sweat gland body to the sweat duct does not reach the sweat duct of the mucus layer level, and that positive components appear when the sweat secretion is continued until the sweat duct is filled with sweat to the level of the mucus layer. Recently, Edelberg (1993) partially modified his membrane model, and proposed a new model of intraductal pressure. It is the poral valve model which suggests that, when pressure is applied to the closed duct space between the sweat gland and the electrode on the skin, the pores in the skin open up like valves, and the conductivity of the duct rises sharply, thereby increasing skin conductance. This pressure model is valuable in that it does not assume the presence of a reabsorption membrane in explaining the rapid changes in conductance. In this model, the sequence of events in an EDR can be summarized as follows; A) At first, the sweat duct is empty and its pores are closed. SCRs and SPRs are not observed at this stage. B) Although the duct is partially filled with sweat, the pores are still closed. Negative components of SCRs and SPRs begin to emerge at this stage. C) When the duct is filled with sweat, the intraductal pressure becomes higher than the tissue pressure, and spreads the poral valve to induce surface sweating. At the same time, sweat is driven into the deeper corneum of the skin. At this stage, SCRs increase even more, and SPRs turn from negative components to positive components. D) When sweat is released from the skin surface, intraductal pressure falls below the tissue pressure, and pores on the skin surface are closed. Closure of the pores results in sudden decreases in conductance. At this stage, SCRs decrease, and the SPR wave form begins to shift toward the negative side after reaching the peak. The results on SPRs and sweating responses obtained in the current study appear to partially verify this model. This model is attractive, and will become even more refined by verifying studies conducted in the future (Hugdahl, 1995). 
Sweating responses were not observed at all in the presence of SPR negative waves in our study, which contradicts the findings of Wilcott (1962). This contradiction may be attributable to the abovementioned differences in the sensitivity and reliability of the methods employed for measuring sweating. Although we observed the significant correlation between the amplitude of SPR positive components and sweating responses, the coefficient did not show a close correlation $(r=0.53)$. It may be explained by the method of measuring the amplitude of positive components of SPR. Development of a reliable method for measuring distorted diphasic waves remains a problem to be solved in the future.

\section{REFERENCES}

Adams, T., \& Vaughan, J. A. 1965 Human eccrine sweat gland activity and palmar electrical skin resistance. Journal of Applied Physiology, 20, 980-983.

Darrow, C. W. 1932 The relation of galvanic skin reflex recovery curve to reactivity, resistance level, and perspiration. Journal of General Psychology, 7, 261-272.

Darrow, C. W. 1964 The rationale for treating the change in galvanic skin response as a change in conductance. Psychophysiology, 1, 31-38.

Edelberg, R. 1964 Independence of galvanic skin response amplitude and sweat production. Journal of Investigative Dermatology, 42, 443-448.

Edelberg, R. 1972 Electrical activity of the skin: Its measurement and uses in psychophysiology. In N. S. Greenficld \& R. A. Sternbach (Eds.), Handbook of psychophysiology. New York: Holt. Pp.367-418.

Edelberg, R. 1993 Electrodermal mechanisms: A critique of the two-effector hypothesis and a proposed replacement. In J. C. Roy, W. Boucsein, D. C. Fowles, \& J. H. Gruzelier (Eds.), Progress in electrodermal research. New York: Plenum. Pp.7-30.

Edelberg, R., Greiner, T., \& Burch, N. R. 1960 Some membrane properties of the effector in the galvanic skin response. Journal of Applied Physiology, 15, 691-696.
Forbes, T. W., \& Bolles, M. M. 1936 Correlation of the response potentials of the skin with "exciting" and non "exciting" stimuli. Journal of Psychology, 2, 273-285.

Fowles, D. C. 1974 Mechanisms of electrodermal activity. In R. F. Thompson \& M. M. Patterson (Eds.), Bioelectric recording techniques. Part C. New York: Academic Press. Pp.231-271.

Fowles, D. C. 1986 The eccrine system and electrodermal activity. In M. G. H. Coles, E. Donchin, \& S. W. Porges (Eds.), Psychophysiology-systems, processes, and applications. Amsterdam: Elsevier. Pp.51-96.

Hugdahl, K. 1995 Electrodermal activity. In K. Hugdahl, Psychophysiology: the mind-body perspective. Cambridge: Harvard University Press. Pp.101-130.

Lader, M. H., \& Montagu, J. D. 1962 The psychogalvanic reflex: A study of the peripheral mechanism. Journal of Neurology, Neurosurgery and Psychiatry, 25, 126-133.

Richter, C. P. 1927 A study of the electrical skin resistance and psychogalvanic reflex in a case of unilateral sweating. Brain, 50, 216-235

Richter, C. P., \& Woodruff, B. G. 1941 Changes produced by sympathectomy in the electrical resistance of the skin. Surgery, 10, 957-970.

Sugenoya, J., \& Ogawa, T. 1985 Characteristics of central sudomotor mechanism estimated by frequency of sweat expulsions. Japanese Journal of Physiology, 35, 783-794.

Sugenoya, J., Iwase, S., Mano, T., \& Ogawa, T. 1990 Identification of sudomotor activity in cutaneous sympathetic nerves using sweat expulsion as the effector response. European Journal of Applied Physiology, 61, 302-308.

Takagi, K., \& Nakayama, T. 1959 Peripheral effector mechanism of galvanic skin reflex. Japanese Journal of Physiology, 5, 75-80.

Venables, P. H., \& Martin, I. 1967 The relation of palmar sweat gland activity to level of skin potential and conductance. Psychophysiology, 3, 302-311.

Wilcott, R. C. 1958 Correlation of skin resistance and potential. Journal of Comparative and Physiological Psychology, 51, 691-696

Wilcott, R. C. 1962 Palmar skin sweating vs. palmar skin resistance and skin potential. Journal of Comparative and Physiological Psychology, 55, 327-331.

Wilcott, R. C. 1964 The partial independence of skin potential and skin resistance from sweating. Psychophysiology, 1, 55-66. 